\title{
A Dual Mode, Local Equilibrium Relaxation Model for Small Molecule Diffusion in a Glassy Polymer
}

\author{
Juchen Guo and Timothy A. Barbari*,† \\ Department of Chemical and Biomolecular Engineering, University of Maryland, \\ College Park, Maryland 20742
}

Received July 25, 2007; Revised Manuscript Received October 18, 2007

\begin{abstract}
FTIR-ATR (Fourier transform infrared-attenuated total reflectance) spectroscopy and a conventional gravimetric sorption balance were used to study the diffusion of acetonitrile from the vapor phase in glassy cellulose acetate (CA). From the gravimetric experiments, the mass uptake kinetics were well described by a simple Fickian model. However, the experimental data from FTIR-ATR spectroscopy, effectively the concentration at a planar solid boundary, could not be described within the simple Fickian framework. A time delay in the measured concentration at the boundary, longer than expected from the simple Fickian model, strongly suggested that an additional physical phenomenon must be operative. In order to explain the observed behavior, a transport model based on dual mode sorption and total immobilization of the hole population was employed with the assumption of local equilibrium relaxed. The resulting model, incorporating finite hole filling/emptying rates, was able to capture the observed FTIR-ATR data and predict the gravimetric results.
\end{abstract}

\section{Introduction}

Understanding the diffusion of small molecules in polymeric materials is important to applications such as membrane separations, barrier materials, controlled-release of pharmaceuticals, and chemical sensors. Glassy polymers are used in those applications listed above where selectivity is important (separations and sensors, in particular). Below the glass transition temperature, molecular motion of the polymer chains is hindered, and the diffusion coefficient has a much stronger dependence on molecular size than above the glass transition temperature. In addition, the nonequilibrium nature of glassy polymers results in sorption and diffusion behavior that can be considerably different than that observed in rubbery polymers, even when the diffusion mechanism is inherently Fickian in nature.

Over the past two decades, the application of FTIR-ATR (Fourier transform infrared-attenuated total reflectance) spectroscopy to the study of diffusion in polymers has grown steadily. ${ }^{1}$ FTIR - ATR is a powerful technique for characterizing diffusion behavior in polymers because it is an in situ technique that can provide reliable short-time data often missed with conventional techniques and information at the molecular level unavailable with other methods. Although FTIR-ATR spectroscopy has gained acceptance as an alternative measurement technique for diffusion in polymers, the strengths of this technique have yet to be fully exploited. Most of the previous work with this technique primarily focused on either water diffusion in polymers ${ }^{2-11}$ or solute-polymer and solute-solute interactions during diffusion in rubbery polymers. ${ }^{12-17}$ Although case II behavior has been observed with FTIR-ATR ${ }^{13}$ and sensor applications have employed a glassy polymer on the ATR crystal, ${ }^{18,19}$ diffusion in polymers below their glass transition temperatures has not been studied extensively with this technique. The present work is the first part of a detailed investigation to determine whether or not FTIR-ATR spectroscopy adds to our knowledge of transport mechanisms in this class of

\footnotetext{
* Corresponding author. E-mail: tab53@georgetown.edu.

† Current address: Georgetown University, Washington, DC 20057.
}

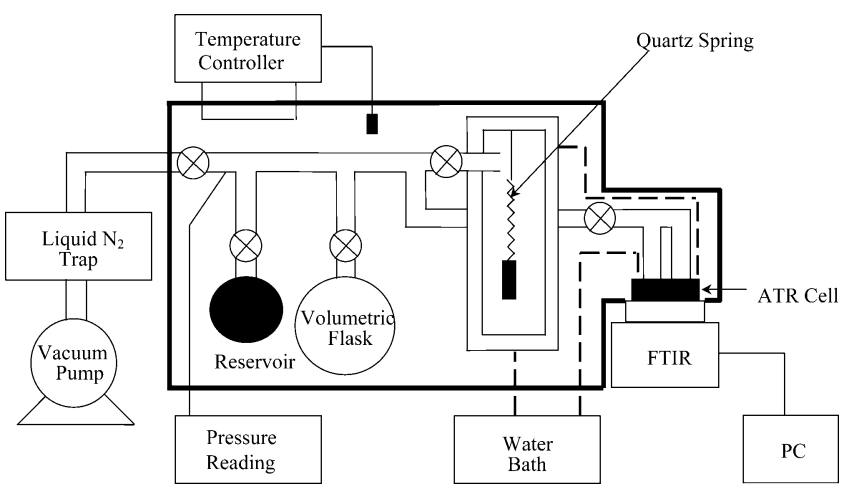

Figure 1. Diagram of the combined vapor generating system, FTIRATR spectrometer, and gravimetric sorption apparatus. ${ }^{15}$

materials. Specifically, FTIR-ATR spectroscopy, combined with a gravimetric sorption balance, was used to examine the sorption and diffusion behavior of acetonitrile in glassy cellulose acetate.

\section{Materials and Methods}

Polymer Film Preparation. Acetonitrile $\left(\mathrm{CH}_{3} \mathrm{CN}\right)$ and cellulose acetate (39.8 wt \% acetyl content, average $M_{\mathrm{n}}$ ca. 30000$)$ were purchased from Sigma-Aldrich and used as received. Cellulose acetate (CA) films were prepared by a solvent-casting method. CA solutions in acetone (purchased from J.T. Baker, 99.5\%, used as received) at a CA concentration of $3.9 \mathrm{wt} \%$ were cast onto the appropriate substrate, either an ATR crystal or a glass coverslip, depending on whether a spectroscopic or gravimetric experiment, respectively, was to be conducted. The substrate was placed in a leveled chamber saturated with acetone. The chamber was sealed at the top by two sheets of FISHERBrand plastic wrap (to control the acetone evaporation rate) and placed in a fume hood for $24 \mathrm{~h}$ at room temperature. After the initial evaporation step, the substrate was taken out of the chamber and placed in a vacuum oven, preheated to $70{ }^{\circ} \mathrm{C}$, for another $24 \mathrm{~h}$. The substrate was then taken out of the oven and immediately placed in a fume hood at room temperature to cool. The dried film on the ATR crystal was found to contain no residual acetone by analysis of the polymer spectrum. Film thickness was measured using a micrometer with $\pm 2.5 \mu \mathrm{m}$ precision. 


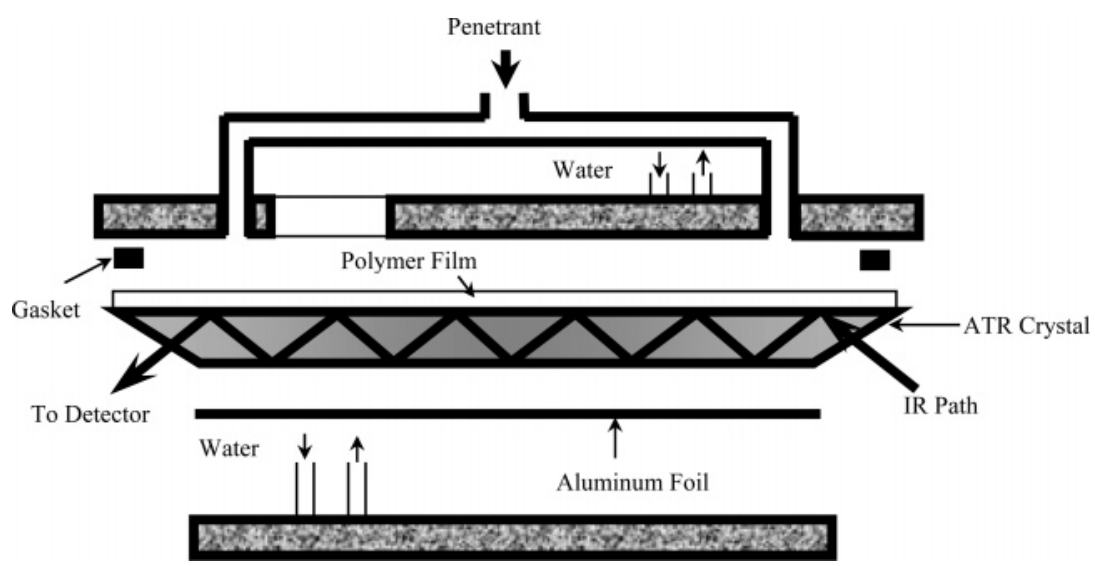

Figure 2. Schematic diagram of the ATR cell. ${ }^{15}$

Experimental Procedure. The sorption and diffusion of acetonitrile from the vapor phase in CA were studied by both gravimetric and spectroscopic techniques. Both types of experiments were conducted with the apparatus shown in Figure 1. The vapor generation system, enclosed by the heavy dark line in Figure 1, is connected independently to a quartz spring microbalance (Ruska Instrument Corp., spring constant of $0.0201 \mathrm{~g} / \mathrm{cm}$ ) and an ATR cell, allowing experiments to be conducted either simultaneously or separately. The vapor pressure of the penetrant is controlled by the heating element inside the insulated chamber, while a temperature-controlled water bath regulates the experimental temperature by circulating water through the glass jacket around the quartz spring and through the ATR cell. Sorption and diffusion measurements were made as a function of activity.

To conduct the gravimetric experiments, the CA polymer films were prepared, as described above, on microscope glass coverslips $(2 \mathrm{~cm} \times 2 \mathrm{~cm})$, removed intact, and hung directly on the quartz spring. The mass uptake was determined from the spring extension, using a cathetometer, as a function of time. Hooke's law, with the predetermined spring constant listed above, $k$, was used to calculate the sorbed mass of acetonitrile, $m_{s}$. Hooke's law must be modified to correct for buoyancy to obtain a precise value of the mass uptake. The corrected form is

$$
m_{\mathrm{s}}=k\left(x-x_{0}\right)+\rho_{\mathrm{g}}\left(V_{\text {sample }}+V_{\text {spring }}\right)
$$

where $x$ is the equilibrium spring extension [cm], $x_{0}$ is the initial extension [cm], $\rho_{\mathrm{g}}$ is the density of the acetonitrile vapor, calculated from an appropriate equation of state $\left[\mathrm{g} / \mathrm{cm}^{3}\right]$, and $V_{\text {sample }}$ and $V_{\text {spring }}$ are the volumes of the sample and spring, respectively $\left[\mathrm{cm}^{3}\right]$.

Infrared spectra for the diffusion experiments were obtained with a Mattson Research Series 1 FTIR spectrometer with a horizontal, temperature-controlled, ATR cell (shown in Figure 2) purchased from Graseby Specac, Inc. The ATR element crystal (Graseby Specac, Inc.) used in this study was a zinc selenide trapezoid (70 $\times 10 \times 6 \mathrm{~mm}$ ) with entry and exit faces beveled at a $45^{\circ}$ angle of incidence for the IR beam and with a refractive index of 2.43 . Experimental spectra are acquired when infrared radiation enters one side of the ATR crystal (clamped in the ATR cell), reflects multiple times down the length of the crystal, is attenuated by infrared absorption at the crystal-polymer interface, and is detected at the opposite side of the ATR crystal with a liquid nitrogen-cooled, mercury-cadmium-telluride (MCT) detector. A background spectrum of the pure polymer on the ATR crystal was subtracted from all spectra obtained during the diffusion process.

The activity was calculated from the ratio of the system pressure $\left(P_{\mathrm{M}}\right)$ to the saturated vapor pressure $\left(P_{\text {sat }}\right)$ of acetonitrile at the system temperature. Prior to each experiment, vacuum is applied to the vapor generation system with the acetonitrile reservoir valve closed (see Figure 1). After the system is evacuated, the reservoir valve is opened and the system is filled with acetonitrile vapor. When the system pressure reading reaches the desired value, the vapor source valve is closed. Once the pressure and temperature inside the vapor generation system reach equilibrium, the valve separating the system from either the ATR cell or the quartz spring is opened. The total amount of acetonitrile in the vapor generation system is much larger than the amount sorbed by the polymer at equilibrium. As a result, the system can be treated as an infinite reservoir of penetrant and the concentration of acetonitrile at the film surface can be assumed constant. The temperature of the ATR cell and gravimetric sorption column was kept at $25{ }^{\circ} \mathrm{C}$ using a water bath for all experiments. The Antoine equation was used to determine the saturated vapor pressure for acetonitrile. ${ }^{20}$

\section{Transport Models}

Simple Fickian Diffusion. For one-dimensional molecular diffusion in a polymer film of thickness $2 L$ ( $-L$ to $L$ ) and a constant (or concentration-averaged) diffusion coefficient, the continuity equation of the diffusing species is described by the following expression:

$$
\frac{\partial C}{\partial t}=D \frac{\partial^{2} C}{\partial z^{2}}
$$

where $C$ is the penetrant concentration and $D$ is the diffusion coefficient. With the initial condition

$$
t=0(\text { all } z): \quad C=0
$$

and the boundary conditions

$$
\begin{aligned}
z=L(t>0): & C=C_{L} \\
z=0(t>0): & \frac{\partial C}{\partial z}=0
\end{aligned}
$$

the following solution to eq 2 is obtained: ${ }^{21}$

$$
\begin{aligned}
\frac{C}{C_{L}}=1-\frac{4}{\pi_{n}} \sum_{0}^{\infty} \frac{(-1)^{n}}{2 n+1} \times & \\
& \quad \exp \left[\frac{-D(2 n+1)^{2} \pi^{2} t}{4 L^{2}}\right] \cos \left[\frac{(2 n+1) \pi z}{2 L}\right]
\end{aligned}
$$

In gravimetric sorption experiments, the mass uptake of sorbed penetrant is measured as a function of time. The ratio of mass uptake to its equilibrium value is obtained by integrating eq 3 over the thickness of the film ( $-L$ to $L$ ). The result of this integration is 


$$
\frac{M_{t}}{M_{\mathrm{eq}}}=1-\sum_{n=0}^{\infty} \frac{8}{(2 n+1)^{2} \pi^{2}} \exp \left[\frac{-D(2 n+1)^{2} \pi^{2} t}{4 L^{2}}\right]
$$

where $M_{t}$ is the mass sorbed at time $t$ and $M_{\mathrm{eq}}$ is the mass sorbed at equilibrium.

In FTIR-ATR diffusion experiments, a polymer film of thickness $L$ is attached to the surface of an ATR crystal and has one surface exposed to the penetrant. With assumptions of weak infrared absorption and constant polymer refractive index, an analogous expression to eq 4 can be derived in terms of absorbance: ${ }^{1,12}$

$$
\begin{aligned}
& \frac{A_{t}}{A_{\mathrm{eq}}}=1-\frac{8 \gamma}{\pi[1-\exp (-2 \gamma L)]} \times \\
& \sum_{n=0}^{\infty}\left[\frac{\exp (g)\left[f \exp (-2 \gamma L)+(-1)^{n}(2 \gamma)\right]}{(2 n+1)\left(4 \gamma^{2}+f^{2}\right)}\right]
\end{aligned}
$$

where

$$
\begin{gathered}
g=\frac{-D(2 n+1)^{2} \pi^{2} t}{4 L^{2}} \\
f=\frac{(2 n+1) \pi}{2 L}
\end{gathered}
$$

and

$$
\gamma=\frac{2 n_{1} \pi\left(\sin ^{2} \theta_{c}-\left(n_{2} / n_{1}\right)^{2}\right)^{1 / 2}}{\lambda}
$$

$\gamma$ is the evanescent field decay coefficient, $n_{1}$ and $n_{2}$ are the refractive indices of the ATR crystal and polymer, respectively, $\theta$ is the incidence angle, and $\lambda$ is the wavelength of absorbed light. The reciprocal of $\gamma$ is often referred to as the depth of penetration, $d_{\mathrm{p}}$, or the characteristic length scale over which absorbance occurs in FTIR-ATR spectroscopy. If $d_{\mathrm{p}} \leq 0.1 L$ (thick-film approximation), then eq 5 can be approximated by ${ }^{15}$

$\frac{A_{t}}{A_{\text {eq }}}=\frac{C_{z=0}}{C_{L}}=1-\frac{4}{\pi_{n}} \sum_{n=0}^{\infty} \frac{(-1)^{n}}{2 n+1} \exp \left[\frac{-D(2 n+1)^{2} \pi^{2} t}{4 L^{2}}\right]$

Dual Mode Sorption and Diffusion. For small molecules at low concentrations in glassy polymers, the most widely accepted model for sorption and diffusion, the dual mode model, assumes that there are two populations of molecules in the polymer: those "dissolved" in the equilibrium portion of the matrix and those residing in the "holes" created by the additional free volume in the system. A key assumption in the application of this model to diffusion is that local equilibrium exists between the two populations. Physically, the two types of sorption mechanisms can be described as follows: ${ }^{22-25}$ (1) Ordinary dissolution in the continuous amorphous matrix. The concentration of this population, $C_{\mathrm{D}}$, is related to pressure (or activity) by Henry's law. (2) Sorption in a limited number of fixed preexisting microvoids, or "holes", in the polymer. The concentration of this population, $C_{\mathrm{H}}$, is related to pressure (or activity) by a Langmuir expression. The dual mode sorption model then gives the total concentration, $C_{\mathrm{T}}$, of sorbed penetrant:

$$
C_{\mathrm{T}}=C_{\mathrm{D}}+C_{\mathrm{H}}=k_{\mathrm{D}} a+C_{\mathrm{H}}^{\prime} \frac{b a}{1+b a}
$$

where $k_{\mathrm{D}}$ is the Henry's law constant, $a$ is the penetrant activity in the gas or vapor phase, $C_{H}^{\prime}$ is the Langmuir capacity constant, and $b$ is the Langmuir affinity constant.

The diffusion of small molecules in glassy polymers at low concentrations is typically represented as Fickian and assumes that the two penetrant species are mobile, with a fraction of the "hole" population immobilized. According to this partial immobilization hypothesis, a fraction, $F$, of the "hole" population is mobile with the same diffusion coefficient as the "dissolved" population, represented by $D_{\mathrm{D}}$. Under these assumptions, species continuity equations can be written for each population. The most general form of the dual mode transport model is ${ }^{26,27}$

$$
\begin{gathered}
\frac{\partial C_{\mathrm{D}}}{\partial t}=\frac{\partial}{\partial z}\left(D_{\mathrm{D}} \frac{\partial C_{\mathrm{D}}}{\partial z}\right)-k_{\mathrm{f}} C_{\mathrm{D}}\left(C_{\mathrm{H}}^{\prime}-C_{\mathrm{H}}\right)+k_{\mathrm{r}} C_{\mathrm{H}} \\
\frac{\partial C_{\mathrm{H}}}{\partial t}=\frac{\partial}{\partial z}\left(F D_{\mathrm{D}} \frac{\partial C_{\mathrm{H}}}{\partial z}\right)+k_{\mathrm{f}} C_{\mathrm{D}}\left(C_{\mathrm{H}}^{\prime}-C_{\mathrm{H}}\right)-k_{\mathrm{r}} C_{\mathrm{H}}
\end{gathered}
$$

where $k_{\mathrm{f}}$ is the rate constant for hole filling and $k_{\mathrm{r}}$ is the rate constant for hole emptying in the Langmuir framework presented in eq 7. Alternatively, the product $F D_{\mathrm{D}}$ can be written as $D_{\mathrm{H}}$, which is interpreted as a diffusion coefficient for molecules sorbed into the "holes", all of which are assumed to be mobile with a lower diffusivity. The initial and boundary conditions are

$$
\begin{gathered}
t=0(\text { all } z): \quad C_{\mathrm{D}}=0, C_{\mathrm{H}}=0 \\
z=0(t>0): \quad \frac{\partial C_{\mathrm{D}}}{\partial z}=0, \frac{\partial C_{\mathrm{H}}}{\partial z}=0 \\
z=L(t>0): \quad C_{\mathrm{D}}=C_{\mathrm{T}_{L}}-C_{\mathrm{H}}, C_{\mathrm{H}}=C_{\mathrm{H}}^{\prime} \frac{b a}{1+b a}
\end{gathered}
$$

where $C_{\mathrm{T}_{L}}$ is the total concentration at $z=L$.

Alternatively, a continuity equation can be written for the total amount of penetrant:

$$
\frac{\partial C_{\mathrm{T}}}{\partial t}=-\frac{\partial N_{\mathrm{T}}}{\partial z}
$$

where $N_{\mathrm{T}}$ is the flux given by

$$
N_{\mathrm{T}}=-D_{\mathrm{D}}\left[\frac{\partial C_{\mathrm{D}}}{\partial z}+F \frac{\partial C_{\mathrm{H}}}{\partial z}\right]
$$

which can be rearranged to

$$
N_{\mathrm{T}}=-D_{\mathrm{D}}\left[1+F \frac{\mathrm{d} C_{\mathrm{H}}}{\mathrm{d} C_{\mathrm{D}}}\right] \frac{\partial C_{\mathrm{D}}}{\partial z}
$$

Assuming local equilibrium between the two populations

$$
k_{\mathrm{f}} C_{\mathrm{D}}\left(C_{\mathrm{H}}^{\prime}-C_{\mathrm{H}}\right)=k_{\mathrm{r}} C_{\mathrm{H}}
$$

$C_{\mathrm{H}}$ can be written in terms of $C_{\mathrm{D}}: 28,29$

$$
C_{\mathrm{H}}=\frac{K C_{\mathrm{D}}}{1+\alpha C_{\mathrm{D}}}
$$

where

$$
K=\frac{C^{\prime}{ }_{\mathrm{H}} b}{k_{\mathrm{D}}}=\frac{C_{\mathrm{H}_{\mathrm{f}}}^{\prime} k_{\mathrm{f}}}{k_{\mathrm{r}}}
$$




$$
\alpha=\frac{b}{k_{\mathrm{D}}}=\frac{k_{\mathrm{f}}}{k_{\mathrm{r}}}
$$

From the local equilibrium assumption, eq 14, one can obtain

$$
\frac{\mathrm{d} C_{\mathrm{H}}}{\mathrm{d} C_{\mathrm{D}}}=\frac{K}{\left(1+\alpha C_{\mathrm{D}}\right)^{2}}
$$

Combining eqs 12 and 17 gives

$$
N_{\mathrm{T}}=-D_{\mathrm{D}}\left[1+\frac{F K}{\left(1+\alpha C_{\mathrm{D}}\right)^{2}}\right] \frac{\partial C_{\mathrm{D}}}{\partial z}
$$

Using eq 18 in eq 10 requires the following relationship:

$$
\frac{\partial C_{\mathrm{T}}}{\partial z}=\frac{\partial C_{\mathrm{D}}}{\partial z}+\frac{\partial C_{\mathrm{H}}}{\partial z}=\left[1+\frac{\mathrm{d} C_{\mathrm{H}}}{\mathrm{d} C_{\mathrm{D}}}\right] \frac{\partial C_{\mathrm{D}}}{\partial z}
$$

Combining eqs 17 and 19 gives

$$
\frac{\partial C_{\mathrm{T}}}{\partial z}=\left[1+\frac{K}{\left(1+\alpha C_{\mathrm{D}}\right)^{2}}\right] \frac{\partial C_{\mathrm{D}}}{\partial z}
$$

Combining eqs 18 with 20 gives

$$
N_{\mathrm{T}}=-D_{\mathrm{D}}\left[\frac{1+\frac{F K}{\left(1+\alpha C_{\mathrm{D}}\right)^{2}}}{1+\frac{K}{\left(1+\alpha C_{\mathrm{D}}\right)^{2}}} \frac{\partial C_{\mathrm{T}}}{\partial z}\right]
$$

Lastly, combining eqs 10 and 21 , the continuity equation in terms of the total concentration for the dual mode model, assuming local equilibrium and a constant (or concentrationaveraged) $D_{\mathrm{D}}$, can be obtained:

$$
\frac{\partial C_{\mathrm{T}}}{\partial t}=D_{\mathrm{D}} \frac{\partial}{\partial z}\left[\frac{1+\frac{F K}{\left(1+\alpha C_{\mathrm{D}}\right)^{2}}}{1+\frac{K}{\left(1+\alpha C_{\mathrm{D}}\right)^{2}}} \frac{\partial C_{\mathrm{T}}}{\partial z}\right]
$$

where

$$
C_{\mathrm{D}}=\frac{-(1+K)+\left[(1+K)^{2}+4 \alpha C_{\mathrm{T}}\right]^{1 / 2}}{2 \alpha}
$$

\section{Results and Discussion}

Converting Infrared Absorbance to Concentration. In order to convert FTIR-ATR absorbance $\left(A_{t}\right)$ to concentration $\left(C_{z=0}\right)$ for application in a transport model, a relationship between the two must be found. A series of FTIR-ATR and gravimetric experiments were done simultaneously to obtain the equilibrium integrated absorbance $\left(\mathrm{cm}^{-1}\right)$ and concentration ( $\left.g_{\text {nitrile }} / g_{\text {polymer }}\right)$, respectively, at different activities $(0.06-0.5)$. The functional group of interest in this study is the $\mathrm{C} \equiv \mathrm{N}$ stretching mode associated with acetonitrile. This region was integrated using a numerical peak integration algorithm in WinFIRST (Mattson Instruments, Inc.) over the wavenumber range of $2350-2200 \mathrm{~cm}^{-1}$. At each activity, the experiment was carried out for $24 \mathrm{~h}$ to ensure equilibrium sorption prior to increasing the pressure to increase the activity. The films were prepared so that the time scale for diffusion $\left(L^{2} / D\right)$ would be the same for both techniques. A linear relationship $\left(C_{z=0}=\epsilon A_{t}\right)$ (a)

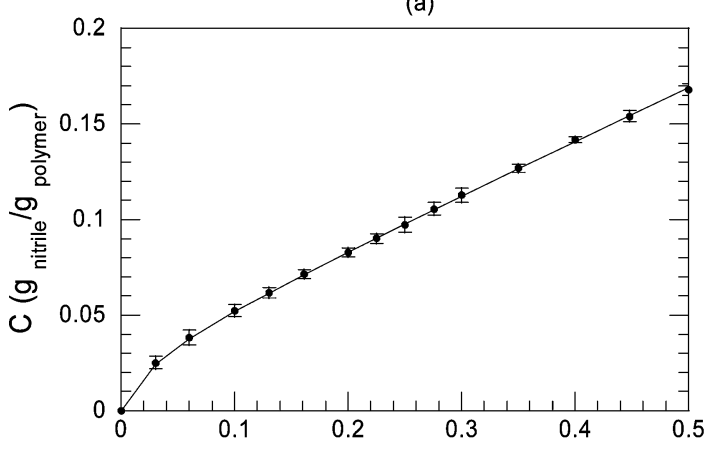

(b)

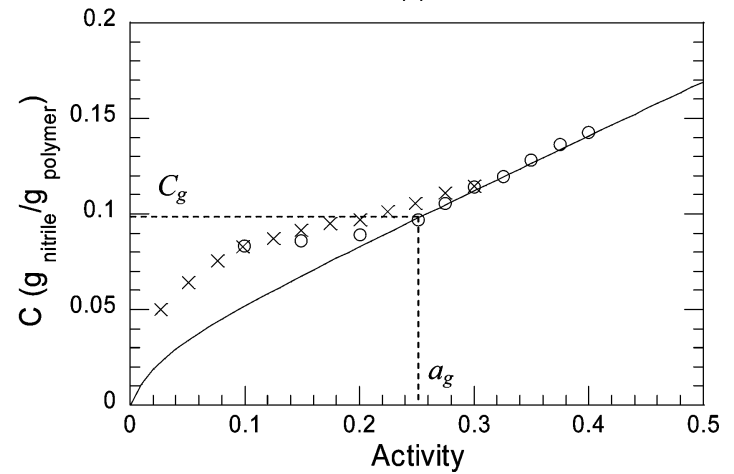

Figure 3. (a) Equilibrium concentration vs activity for acetonitrile in $\mathrm{CA}$ at $25^{\circ} \mathrm{C}$ and the dual mode sorption model fit of the data (solid line). (b) Desorption hysteresis. The solid line is the sorption curve from (a); circles and crosses are desorption isotherms starting from activities 0.4 and 0.3 , respectively.

between ATR absorbance and concentration was obtained with $\epsilon=0.0873\left[g_{\text {nitrile }} /\left(g_{\text {polymer }}{ }^{\circ} \mathrm{cm}\right)\right]\left(r^{2}=0.9959\right)$. The depth of penetration for this system was calculated to be $7.9 \times 10^{-5}$ $\mathrm{cm}$, which is $20-50$ times less than the film thicknesses used in this study. Therefore, it was reasonable to use the thick film approximation, eq 6 , and equate the measured absorbance to $C_{z=0}$.

Sorption Isotherm and Desorption Hysteresis for Acetonitrile in Cellulose Acetate. In two separate series of experiments with freshly cast CA films, FTIR-ATR absorbances at equilibrium were obtained as a function of activity, from 0.06 to 0.5 . The absorbance data thus obtained were converted to equilibrium concentrations using the value of $\epsilon$ reported above and the average at each activity was used to obtain the sorption isotherm in Figure 3a. The dual mode sorption model, eq 7, was used to fit the data to obtain the dual mode sorption parameters: $k_{\mathrm{D}}=0.282\left(g_{\text {nitrile }} / g_{\text {polymer }}\right) ; C_{\mathrm{H}}^{\prime}=0.030\left(g_{\text {nitrile }} /\right.$ $\left.g_{\text {polymer }}\right) ; b=38.1$.

The relatively low value of $C_{\mathrm{H}}^{\prime}$ and the long range of activity over which the isotherm is linear suggest that the CA may undergo a concentration-induced glass transition at some intermediate activity. Since the focus of this work is an investigation of glassy state diffusion using FTIR-ATR, desorption isotherms were measured to determine whether such a transition is present. CA was exposed to acetonitrile vapor at an activity of either 0.4 or 0.3 , until the equilibrium mass uptake was reached. The resulting desorption isotherms for each initial activity are plotted in Figure $3 \mathrm{~b}$ alongside the dual mode sorption isotherm.

It can be seen that the desorption isotherm starting from activity 0.4 initially follows the sorption isotherm, departing near activity 0.25 . It is well-known that, for rubbery polymers, the sorption and desorption isotherms follow the same curve. ${ }^{30,31}$ 


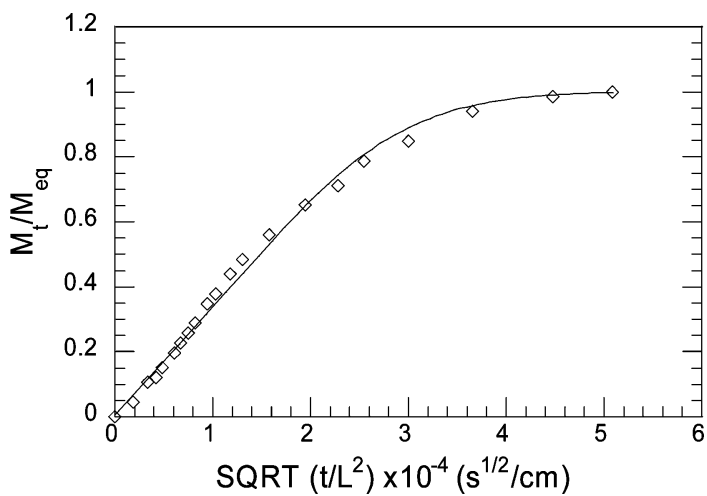

Figure 4. Mass uptake kinetics and the fit using eq 4 at activity 0.06 . $L=30.2 \mu \mathrm{m}$ and $T=25^{\circ} \mathrm{C}$.

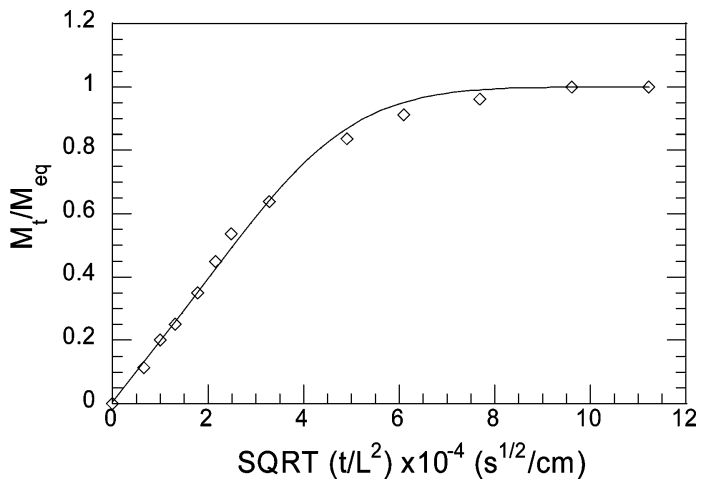

Figure 5. Mass uptake kinetics and the fit using eq 4 at activity 0.1 . $L=28.6 \mu \mathrm{m}$ and $T=25^{\circ} \mathrm{C}$.

Furthermore, it is also well-established that desorption hysteresis is a characteristic of the glassy state, and the onset of this hysteresis is identified as the glass transition concentration. ${ }^{30}$ This behavior suggests that the acetonitrile/CA system is in a rubbery state at activity 0.4 , and a penetrant-induced glass transition occurs near activity 0.25 . The desorption isotherm starting from activity 0.3 shows a more immediate departure from the sorption isotherm but ultimately follows the desorption isotherm generated from activity 0.4 . Since the glass transition is itself history dependent, these results suggest that the polymer at activity 0.3 may not have completely relaxed to a rubbery state, even though the sorption is considered to be at equilibrium, over the time allowed for the measurement. On the basis of the desorption isotherm starting at activity 0.4 and assuming that the polymer is fully relaxed at this activity, the glass transition is taken to be at activity 0.25 . The glass transition concentration corresponding to activity 0.25 is $C_{\mathrm{g}}=0.0977\left(g_{\text {nitrile }} / g_{\text {polymer }}\right)$. Diffusion experiments were therefore limited in this work to activities of 0.25 and lower.

Simple Fickian Diffusion. Gravimetric measurements were made at activities of $0.06,0.1$, and 0.2 , and the data were fit using eq 4. Representative examples of the time-evolved mass uptake data and corresponding fits are shown in Figures 4, 5 and 6 , respectively. The linear dependence at short times between mass uptake and the square root of time clearly shows that the underlying physical mechanism for diffusion in the acetonitrile-CA system is Fickian. Experiments at each activity were done in triplicate. The diffusion coefficients are listed in Table 1.

Similar experiments were performed with the spectroscopic technique as a function of activity. FTIR-ATR diffusion experiments were conducted at activities of $0.06,0.1,0.15,0.2$, and 0.25 . Infrared spectra at a few time points for acetonitrile diffusion in CA at activity 0.2 and a thickness $L$ of $49.2 \mu \mathrm{m}$

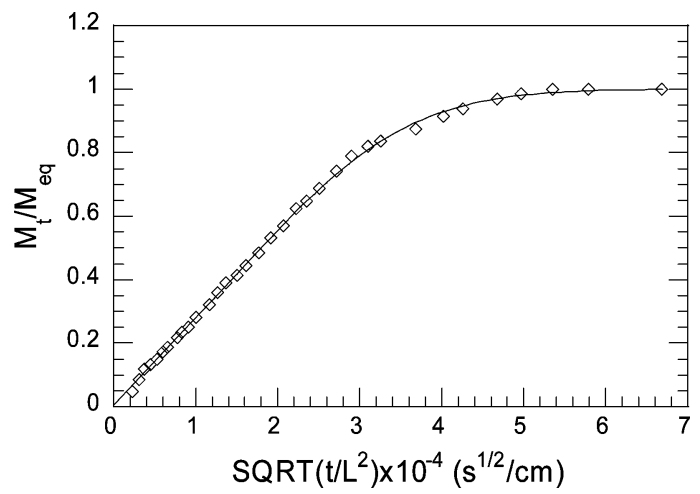

Figure 6. Mass uptake kinetics and the fit using eq 4 at activity 0.2 . $L=50.4 \mu \mathrm{m}$ and $T=25^{\circ} \mathrm{C}$.

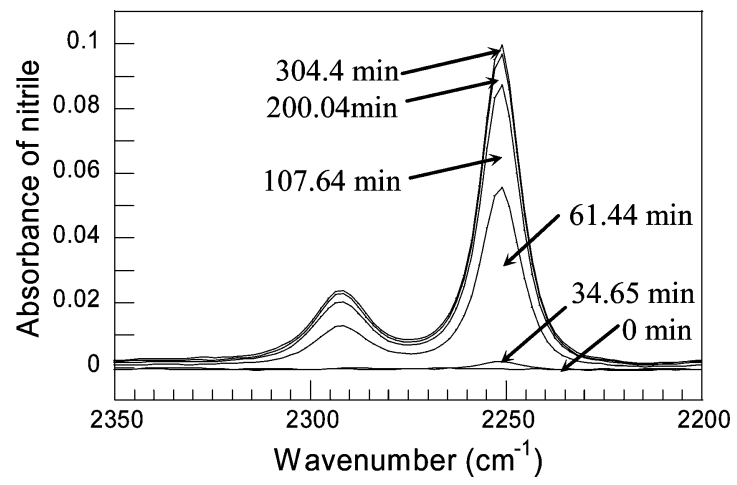

Figure 7. Time-resolved acetonitrile absorbance spectra in cellulose acetate at activity of 0.20 and $T=25^{\circ} \mathrm{C}$.

Table 1. Diffusion Coefficients from the Dual Mode, Local Equilibrium Relaxation Model $\left(k_{\mathrm{r}}=4.85 \times 10^{-3} \mathrm{~s}^{-1}\right)$, and Diffusion Coefficients from Gravimetric Experiments

\begin{tabular}{ccc}
\hline $\begin{array}{c}\text { activity } \\
(N=3)\end{array}$ & $\begin{array}{c}D_{\mathrm{D}} \times 10^{10} \mathrm{~cm}^{2} / \mathrm{s} \\
\text { from IR experiments }\end{array}$ & $\begin{array}{c}D \times 10^{10} \mathrm{~cm}^{2} / \mathrm{s} \\
\text { from mass uptake }\end{array}$ \\
\hline 0.06 & $1.91 \pm 0.03$ & $2.06 \pm 0.26$ \\
0.10 & $2.96 \pm 0.02$ & $3.20 \pm 0.42$ \\
0.15 & $4.66 \pm 0.01$ & \\
0.20 & $6.38 \pm 0.04$ & $6.50 \pm 0.40$ \\
0.25 & $7.04 \pm 0.11$ &
\end{tabular}

are shown in Figure 7. The integrated absorbances, normalized by the equilibrium absorbance, are plotted in Figure 8. In Figure $8 \mathrm{a}$, eq 6 is used to calculate the concentration at the interface $\left(C_{z=0}\right)$ as a function of time using the average diffusion coefficient obtained from mass uptake at this activity, $D=6.5$ $\times 10^{-10} \mathrm{~cm}^{2} / \mathrm{s}$. The predicted values of concentration at the interface are consistently higher than the values measured spectroscopically. In Figure 8b, eq 6 was used to obtain the best fit to the data, resulting in a diffusion coefficient of 6.25 $\times 10^{-10} \mathrm{~cm}^{2} / \mathrm{s}$; however, the fit is rather poor. It is clear that there is a time delay in the spectroscopic data that cannot be captured by a simple Fickian model.

Dual Mode with Local Equilibrium. One explanation for the observed lag in the concentration at the polymer-crystal interface is that immobilization of acetonitrile in "holes" removes a fraction of penetrant from the diffusion process, thereby lowering local gradients and decreasing the flux. Assuming local equilibrium between the two populations, the spectroscopic data for the same experiment at activity 0.2 were fit using eq 22 in conjunction with a numerical simulation of the differential equation. If $F$, the fraction of the "hole" population that is mobile, is taken to be zero, then this would remove the most penetrant molecules from the process. The best fit value of $D_{\mathrm{D}}$ for eq 22 assuming $F=0$ was $2.20 \times 10^{-10}$ 


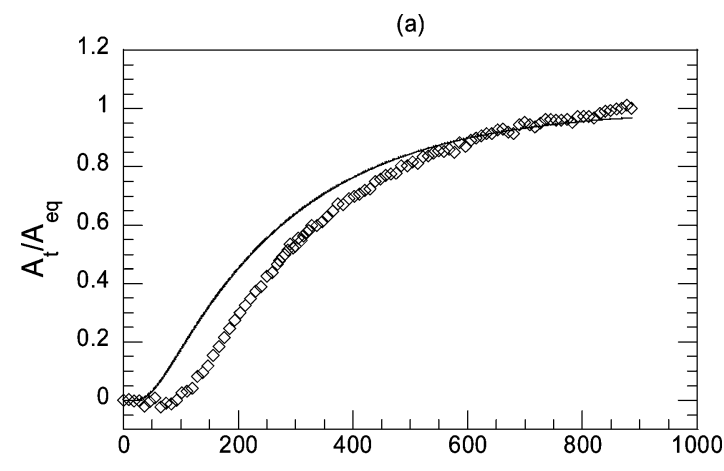

(b)

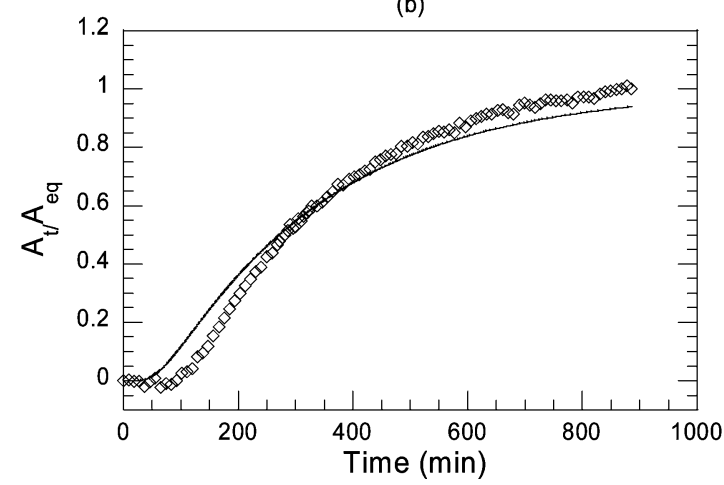

Figure 8. FTIR-ATR absorbance data of acetonitrile in CA at activity 0.2 and $T=25{ }^{\circ} \mathrm{C}$ with (a) the calculated absorbance curve using the simple Fickian model (eq 6 ) and the diffusion coefficient obtained from the gravimetric measurements and (b) the best fit simple Fickian model (eq 6). $L=49.2 \mu \mathrm{m}$.

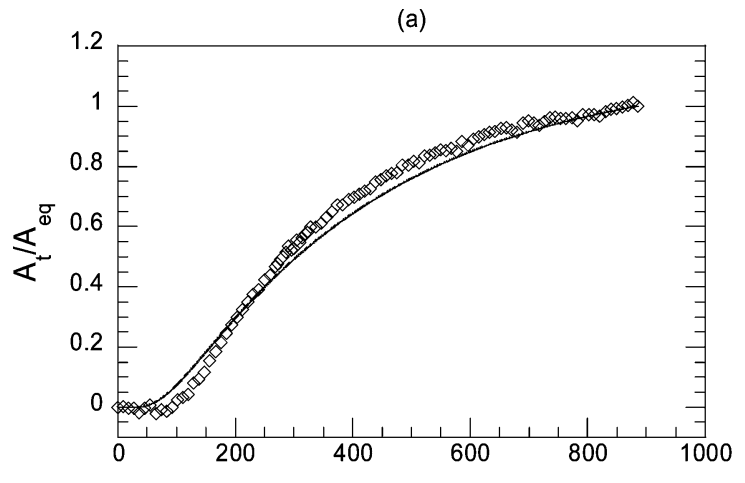

(b)

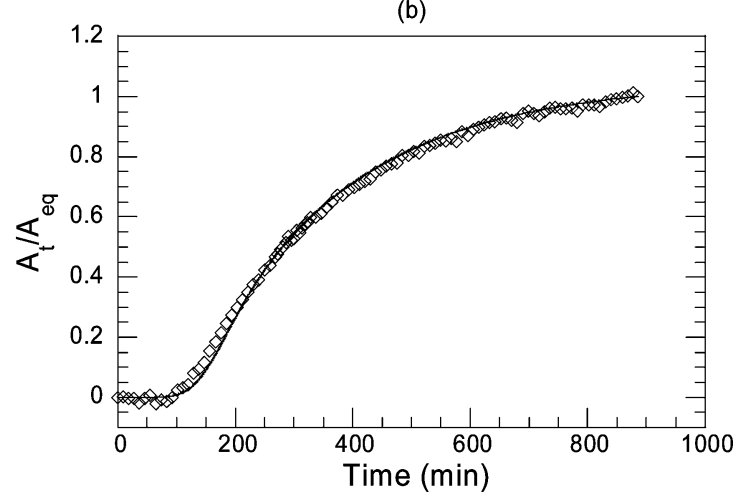

Figure 9. FTIR-ATR absorbance data of acetonitrile in CA at activity 0.2 and $T=25{ }^{\circ} \mathrm{C}$ with (a) the best fit using the dual mode model under local equilibrium assumption, eq 22, and (b) the best fit using the dual mode model with the local equilibrium assumption relaxed. $L$ $=49.2 \mu \mathrm{m}$.

$\mathrm{cm}^{2} / \mathrm{s}$, and the resulting fit is shown in Figure 9a. As was the case for simple Fickian diffusion, the dual mode model with local equilibrium cannot reproduce the time delay in concentration at the polymer-crystal interface. There appears to be another mechanism operative during diffusion in a glassy polymer that results in this delay. The same phenomenon occurs at all activities, as will be shown below, and is reproducible at any given activity.

Local Equilibrium Relaxation. If the local equilibrium assumption is relaxed, the rates of hole filling and hole emptying, given in the generalized dual mode transport model of eqs 8 and 9 , become finite, and the hole filling/emptying mechanism can further slow the diffusion of the mobile species. A numerical method was used to solve these equations, which was then coupled to a fitting routine to determine the best fit values for the relevant adjustable parameters in the model. Equations 8 and 9 were transformed into nondimensional form as follows (assuming a constant, or concentration-averaged, $D_{\mathrm{D}}$ ):

$$
\begin{gathered}
\frac{\partial \theta_{\mathrm{D}}}{\partial \tau}=\frac{\partial^{2} \theta_{\mathrm{D}}}{\partial \xi^{2}}-\phi \theta_{\mathrm{D}}\left(\theta_{\mathrm{H}}^{\prime}-\theta_{\mathrm{H}}\right)+\theta_{\mathrm{H}} \\
\frac{\partial \theta_{\mathrm{H}}}{\partial \tau}=F \frac{\partial^{2} \theta_{\mathrm{H}}}{\partial \xi^{2}}+\phi \theta_{\mathrm{D}}\left(\theta_{\mathrm{H}}^{\prime}-\theta_{\mathrm{H}}\right)-\theta_{\mathrm{H}}
\end{gathered}
$$

where

$$
\begin{gathered}
\theta_{\mathrm{D}}=\frac{C_{\mathrm{D}}}{C_{\mathrm{T}_{L}}} \\
\theta_{\mathrm{H}}=\frac{C_{\mathrm{H}}}{C_{\mathrm{T}_{L}}} \\
\tau=t k_{\mathrm{r}} \\
\xi=\frac{z}{\sqrt{D_{\mathrm{D}} / k_{\mathrm{r}}}} \\
k_{\mathrm{f}} C_{\mathrm{T}_{L}}=\frac{b C_{\mathrm{T}_{L}}}{k_{\mathrm{r}}}=\frac{k_{\mathrm{D}}}{\theta_{\mathrm{H}}^{\prime}}=\frac{C_{\mathrm{H}}}{C_{\mathrm{T}_{L}}}
\end{gathered}
$$

The transformed initial and boundary conditions are

$$
\begin{gathered}
\tau=0(\text { all } \xi): \quad \theta_{\mathrm{D}}=0, \theta_{\mathrm{H}}=0 \\
\xi=0(\tau \geq 0): \quad \frac{\partial \theta_{\mathrm{D}}}{\partial \xi}=0, \frac{\partial \theta_{\mathrm{H}}}{\partial \xi}=0 \\
\xi=\frac{L}{\sqrt{D_{\mathrm{D}} / k_{\mathrm{r}}}}(\tau \geq 0): \quad \theta_{\mathrm{D}}=1-\theta_{\mathrm{H}}, \theta_{\mathrm{H}}=\theta_{\mathrm{H}}^{\prime} \frac{b a}{1+b a}
\end{gathered}
$$

The ratio of the rate constants, $k_{\mathrm{f}}$ and $k_{\mathrm{r}}$, was fixed at 135.3 ( $\left.g_{\text {polymer }} / g_{\text {nitrile }}\right)$, which is the ratio of $b$ to $k_{\mathrm{D}}$. The value of $C_{\mathrm{H}}^{\prime}$ was fixed from the isotherm at $0.030\left(g_{\text {nitrile }} / g_{\text {polymer }}\right)$. The "hole" population was assumed to be immobile $(F=0)$ because preliminary results using $F$ as an adjustable parameter resulted in values near zero for the best fits. Hence, there are only two adjustable parameters in the nondimensional dual mode transport model presented here: $k_{\mathrm{r}}$ in $\tau$ and $D_{\mathrm{D}} / k_{\mathrm{r}}$ in $\xi$. The result using the dual mode transport model with local equilibrium relaxed for the 0.2 activity data is shown in Figure 9b. The model 


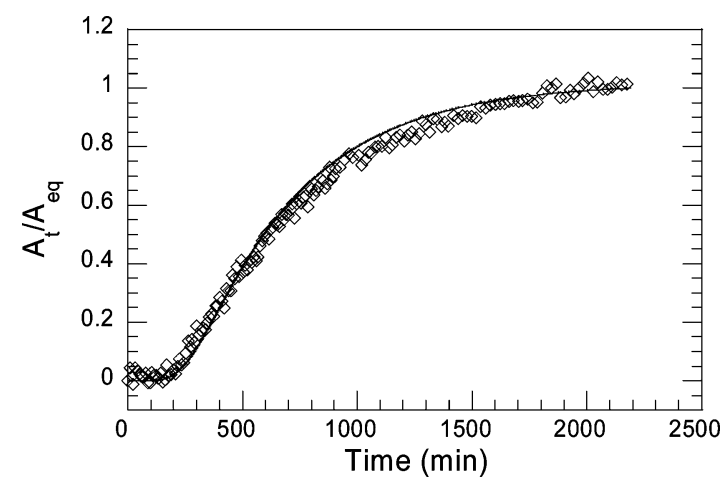

Figure 10. FTIR-ATR data for absorbance of acetonitrile in CA at activity 0.06 with the fit using the dual mode, local equilibrium relaxation model. $T=25^{\circ} \mathrm{C}$ and $L=20.8 \mu \mathrm{m}$.

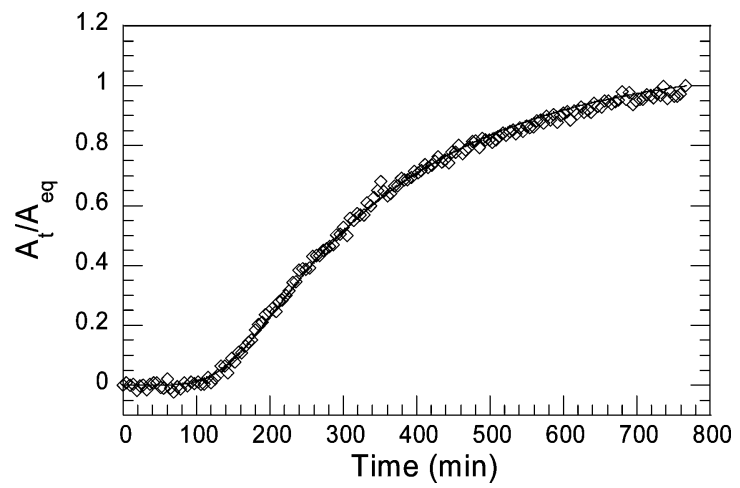

Figure 11. FTIR-ATR data for absorbance of acetonitrile in CA at activity 0.1 with the fit using the dual mode, local equilibrium relaxation model. $T=25{ }^{\circ} \mathrm{C}$ and $L=29.8 \mu \mathrm{m}$.

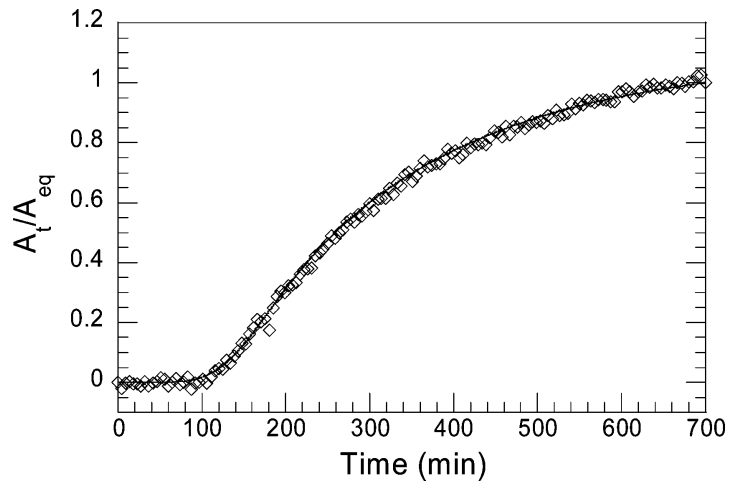

Figure 12. FTIR-ATR data for absorbance of acetonitrile in CA at activity 0.15 with the fit using the dual mode, local equilibrium relaxation model. $T=25^{\circ} \mathrm{C}$ and $L=38.4 \mu \mathrm{m}$.

captures the time delay very well, indicating that accounting for the finite rate at which the holes are filled and emptied better describes the sorption kinetics for acetonitrile in cellulose acetate. From triplicate experiments at activity 0.2 , the mean values of $D_{\mathrm{D}}$ and $k_{\mathrm{r}}$ were determined to be $(6.38 \pm 0.04) \times$ $10^{-10} \mathrm{~cm}^{2} / \mathrm{s}$ and $(4.85 \pm 0.09) \times 10^{-3} \mathrm{~s}^{-1}$, respectively.

To test the robustness of the model and its physical interpretation, the rate constants for hole filling and emptying were assumed to be independent of activity. Fixing the value of $k_{\mathrm{r}}$ at $4.85 \times 10^{-3} \mathrm{~s}^{-1}$, FTIR-ATR data for diffusion at the remaining activities were fit with $D_{\mathrm{D}}$ as the only adjustable parameter. Representative fits for each of these activities are shown in Figures $10-13$, and the mean values of $D_{\mathrm{D}}$ are listed in Table 1.

Prediction of Mass Uptake. As a further validation of the dual mode transport model with local equilibrium relaxation,

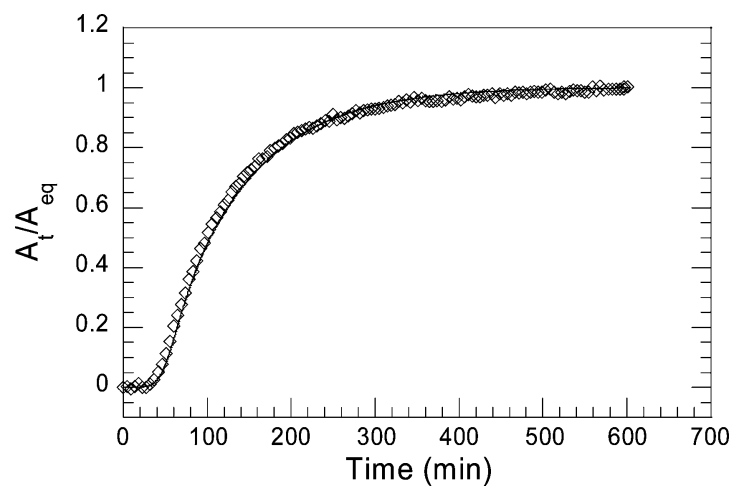

Figure 13. FTIR-ATR data for absorbance of acetonitrile in CA at activity 0.25 with the fit using the dual mode, local equilibrium relaxation model. $T=25^{\circ} \mathrm{C}$ and $L=30.4 \mu \mathrm{m}$.

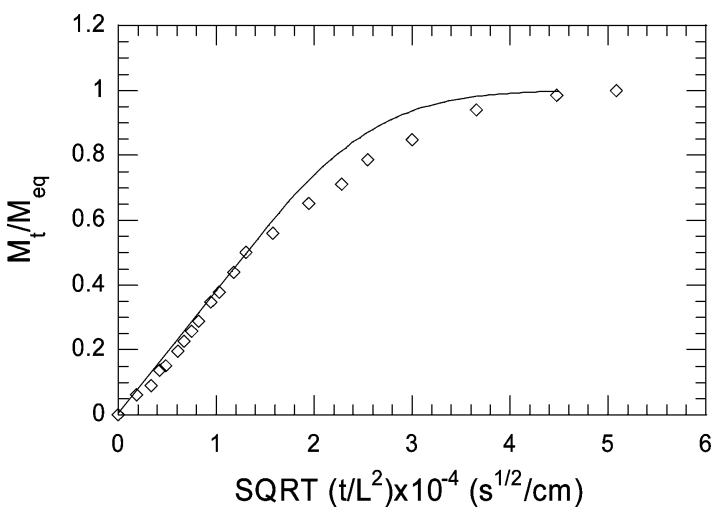

Figure 14. Prediction of mass uptake at activity 0.06 using the dual mode, local equilibrium relaxation model. Open diamonds represent actual mass uptake data. $L=30.2 \mu \mathrm{m}$.

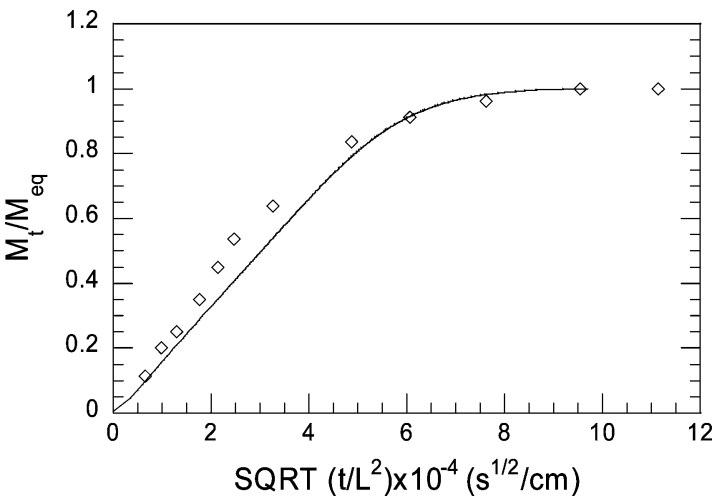

Figure 15. Prediction of mass uptake at activity 0.1 using the dual mode, local equilibrium relaxation model. Open diamonds represent actual mass uptake data. $L=28.6 \mu \mathrm{m}$.

the mass uptake curve was predicted using the parameters obtained from the FTIR-ATR data. First, the total concentration profile was calculated numerically, and then the concentration profile was integrated over the film thickness to obtain the mass uptake. The calculated mass uptake using the mean value of the diffusion coefficient from the IR experiments and the actual mass uptake data are plotted as a function of $\left(t / L^{2}\right)^{1 / 2}$ at activities $0.06,0.1$, and 0.2 in Figures 14, 15, and 16, respectively. In these figures, the open diamonds are the mass uptake experimental data and the solid lines are the simulations from the dual mode transport model with local equilibrium relaxation. In general, there is good agreement between the prediction and the actual data, particularly at the highest activity. 


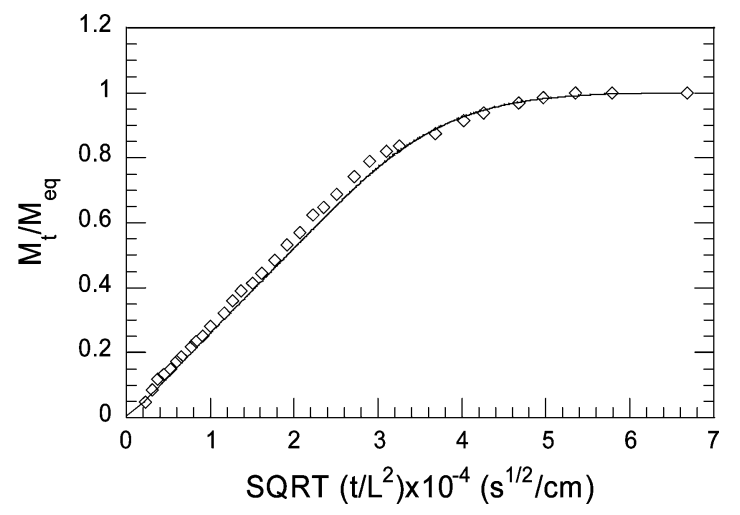

Figure 16. Prediction of mass uptake at activity 0.2 using the dual mode, local equilibrium relaxation model. Open diamonds represent actual mass uptake data. $L=50.4 \mu \mathrm{m}$.

\section{Conclusion}

The results presented here indicate that the assumption of local equilibrium in the dual mode model may not be applicable when describing transient diffusion behavior in a glassy polymer. One of the strengths of FTIR-ATR spectroscopy applied to diffusion in a polymer film is that it effectively measures the concentration at a point, the polymer-crystal interface, provided the film thickness is much larger than the depth of penetration of the evanescent field into the film. Therefore, the technique can be a stringent test of transport models, such as the dual mode model, and uncover phenomena that could be masked when measuring an integrated quantity, such as total mass in gravimetric measurements.

The dual mode, local equilibrium relaxation (DMLER) model describes well the physics of acetonitrile diffusion in glassy cellulose acetate. The model appears rather robust in that the characteristic rate constant for the hole-filling process $\left(k_{\mathrm{r}}\right)$ from one activity can be kept constant as activity is varied, leaving the diffusion coefficient for the dissolved population $\left(D_{\mathrm{D}}\right)$ as the only adjustable parameter. Physically, at low activities, the slight increase in $D_{\mathrm{D}}$ with activity suggests an increase in chain mobility. In contrast, a constant value of $k_{\mathrm{r}}$ suggests that chain mobility around a pre-existing hole does not influence the rate of filling (or emptying) that hole. The observed desorption hysteresis suggests a concentration-induced glass transition, which must be accounted for separately. Future work will address this transition by studying diffusion in this system at activities greater than 0.25 . The dual mode local equilibrium relaxation model will also be challenged with desorption and dilation data in future studies.
Acknowledgment. Financial support for J. Guo from the University of Maryland, College Park, is gratefully acknowledged.

\section{References and Notes}

(1) Elabd, Y. A.; Baschetti, M. G.; Barbari, T. A. J. Polym. Sci., Part B: Polym. Phys. 2003, 41, 2794-2807.

(2) Sammon, C.; Everall, N.; Yarwood, J. Macromol. Symp. 1997, 119 , 189-196.

(3) Sammon, C.; Mura, C.; Hajatdoost, S.; Yarwood, J. J. Mol. Liq. 2002, 69-97, 305-315.

(4) Pereira, M. R.; Yarwood, J. J. Chem. Soc., Faraday Trans. 1996, 92, $2731-2736$

(5) Pereira, M. R.; Yarwood, J. J. Chem. Soc., Faraday Trans. 1996, 92, $2737-2743$.

(6) Hajatdoost, S.; Yarwood, J. J. Chem. Soc., Faraday Trans. 1997, 93, $1613-1620$

(7) Sammon, C.; Mura, C.; Yarwood, J.; Everall, N.; Swart, R.; Hodge, D. J. Phys. Chem. B 1998, 102, 3402-3411.

(8) Sammon, C.; Yarwood, J.; Everall, N. Polymer 2000, 41, 2521-2534.

(9) Mura, C.; Yarwood, J.; Swart, R.; Hodge, D. Polymer 2001, 42, 41414152.

(10) Hajatdoost, S.; Sammon, C.; Yarwood, J. Polymer 2002, 43, 18211827.

(11) Deng, C.; Sammon, C.; Yarwood, J. Polymer 2003, 44, 2057-2065.

(12) Fieldson, G. T.; Barbari, T. A. Polymer 1993, 34, 1146-1153.

(13) Fieldson, G. T.; Barbari, T. A. AIChE J. 1995, 41, 795-804.

(14) Hong, S. U.; Barbari, T. A.; Sloan, J. M. J. Polym. Sci., Part B: Polym. Phys. 1998, 36, 337-344.

(15) Elabd, Y. A.; Barbari, T. A. AIChE J. 2001, 47, 1255-1262.

(16) Elabd, Y. A.; Sloan, J. M.; Tan, N. B.; Barbari, T. A. Macromolecules 2001, 34, 6268-6273.

(17) Elabd, Y. A.; Barbari, T. A. AIChE J. 2002, 48, 1610-1620.

(18) Murphy, B.; McLoughlin, P. Int. J. Environ. Anal. Chem. 2003, 83, 653-662.

(19) Murphy, B.; Kirwan, P.; McLoughlin, P. Anal. Bioanal. Chem. 2003 377, 195-202.

(20) Poling, B. E.; Prausnitz, J. M.; O'Connell, J. P. The Properties of Gases and Liquids, 5th ed.; McGraw-Hill: New York, 2001; p 638.

(21) Crank, J. The Mathematics of Diffusion, 2nd ed.; Clarendon Press: Oxford, 1975; p 47.

(22) Frisch, H. L. Polym. J. 1991, 23, 445-456.

(23) Volkov, V. V. Polym. J. 1991, 23, 457-466

(24) Barrer, R. M. J. Membr. Sci. 1984, 18, 25-35.

(25) Ismail, A. F.; Lorna, W. Sep. Purif. Technol. 2002, 27, 173-194.

(26) Paul, D. R.; Yampol'skii, Yu. P. Polymeric Gas Separation Membranes; CRC Press: Boca Raton, FL, 2000; p 313.

(27) Fredrickson, G. H.; Helfand, E. Macromolecules 1985, 18, 22012207.

(28) Paul, D. R.; Koros, W. J. J. Polym. Sci. 1976, 14, 675-685.

(29) Koros, W. J.; Paul, D. R.; Rocha, A. A. J. Polym. Sci. 1976, 14, 687702.

(30) Kamiya, Y.; Mizoguchi, K.; Naito, Y.; Hirose, T. J. Polym. Sci., Part B: Polym. Phys. 1986, 24, 535-547.

(31) Kamiya, Y.; Mizoguchi, K.; Hirose, T.; Naito, Y. J. Polym. Sci., Part B: Polym. Phys. 1989, 27, 879-892.

MA071662C 


\title{
CORRECTION
}

\section{A Dual Mode, Local Equilibrium Relaxation Model for Small Molecule Diffusion in a Glassy Polymer}

\author{
Juchen Guo and Timothy A. Barbari ${ }^{1}$ * \\ Department of Chemical and Biomolecular Engineering \\ University of Maryland, College Park, MD 20742
}

${ }^{1}$ Current address: Georgetown University, Washington, DC 20057

*Corresponding author. E-mail: tab53@georgetown.edu

In a recent paper (Guo, J; Barbari, T.A. Macromolecules, 2008, 41, 238-245), we relaxed the local equilibrium assumption often employed in the development of transport models that are based on dual mode sorption of small molecules in glassy polymers. The resulting model was then used to fit sorption kinetics data measured by Fourier transform infrared attenuated total reflectance (FTIR-ATR) spectroscopy for acetonitrile in glassy cellulose acetate. The motivation for relaxing this assumption was twofold: (1) while a simple Fickian framework could fit mass uptake (integral) data for this system, it could not capture the lag in the FTIR-ATR (local) data, suggesting a mechanism in addition to diffusion (such as hole filling in the dual mode model) that could slow the transport process; and (2) incorporation of the dual mode sorption model with local equilibrium, while an improvement over the simple Fickian model, did not fully capture the lag in the FTIR-ATR data. Recently, we discovered an error in the equation used to test the local equilibrium assumption in the dual mode framework. Using the correct expression, the dual mode model assuming local equilibrium is able to describe the local concentration data obtained from the spectroscopic technique, negating the need to relax this assumption and introduce an additional parameter.

The dual mode, local equilibrium relaxation model used to fit the data in the original paper was developed and used correctly. Therefore, we feel it is worthwhile to use this opportunity to explain the relevance of the additional kinetic parameter that results from the full model in the context of the local equilibrium assumption and to illustrate the importance of carefully assessing results from optimization tools for fitting purposes. 


\section{Dual Mode Sorption and Transport}

The development that follows is a summary of that found in the original paper and the reader should refer to that source for complete details. The dual mode sorption model assumes that there are two populations of molecules sorbed into a glassy polymer: (1) one dissolved in the dense portion of the polymer (the Henry's law population), and (2) one residing in pre-existing excess free volume or "holes" (the Langmuir population). According to this model, the total concentration, $C_{T}$, of sorbed penetrant is given by:

$C_{T}=C_{D}+C_{H}=k_{D} a+C_{H}^{\prime} \frac{b a}{1+b a}$

where $k_{D}$ is the Henry's law constant, $a$ is the penetrant activity in the gas or vapor phase, $C_{H}^{\prime}$ is the Langmuir capacity constant and $b$ is the Langmuir affinity constant.

At low concentrations, the diffusion of each population is represented as Fickian and one interpretation takes the two penetrant populations as mobile, with a fraction, $F$, of the "hole" population immobilized. The resulting dual mode transport model, in its most general form to account for the hole filling mechanism, is:

$$
\begin{aligned}
& \frac{\partial C_{D}}{\partial t}=\frac{\partial}{\partial z}\left(D_{D} \frac{\partial C_{D}}{\partial z}\right)-k_{f} C_{D}\left(C_{H}^{\prime}-C_{H}\right)+k_{r} C_{H} \\
& \frac{\partial C_{H}}{\partial t}=\frac{\partial}{\partial z}\left(F D_{D} \frac{\partial C_{H}}{\partial z}\right)+k_{f} C_{D}\left(C_{H}^{\prime}-C_{H}\right)-k_{r} C_{H}
\end{aligned}
$$

where $D_{D}$ is the diffusion coefficient, $k_{f}$ is the rate constant for hole filling and $k_{r}$ is the rate constant for hole emptying.

Assuming local equilibrium between the two populations, the model reduces to

$$
\frac{\partial C_{T}}{\partial t}=\frac{\partial}{\partial z}\left(D_{D} \frac{\partial C_{D}}{\partial z}+F D_{D} \frac{\partial C_{H}}{\partial z}\right)
$$

and

$$
k_{f} C_{D}\left(C_{H}^{\prime}-C_{H}\right)=k_{r} C_{H}
$$

Eliminating $C_{H}$, the model can be written, assuming $D_{D}$ constant or concentration averaged, as

$$
\frac{\partial C_{T}}{\partial t}=D_{D} \frac{\partial}{\partial z}\left[\frac{1+\frac{F K}{\left(1+\alpha C_{D}\right)^{2}}}{1+\frac{K}{\left(1+\alpha C_{D}\right)^{2}}} \frac{\partial C_{T}}{\partial z}\right]
$$

where 
$C_{D}=\frac{-\left(1+K-\alpha C_{T}\right)+\left[\left(1+K-\alpha C_{T}\right)^{2}+4 \alpha C_{T}\right]^{\frac{1}{2}}}{2 \alpha}$

$\alpha=\frac{b}{k_{D}}=\frac{k_{f}}{k_{r}}$

$K=\frac{C_{H}^{\prime} b}{k_{D}}$

In the original paper, Equation (7) was incorrectly written as

$C_{D}=\frac{-(1+K)+\left[(1+K)^{2}+4 \alpha C_{T}\right]^{\frac{1}{2}}}{2 \alpha}$,

which was the expression inadvertently used to test the local equilibrium assumption with the FTIRATR data for acetonitrile in cellulose acetate, leading to the conclusion that the full expressions given in Equations (2) and (3) should be used instead, introducing $k_{r}$ as an additional parameter to fit the data.

\section{Model Comparison - Local Equilibrium vs. Local Equilibrium Relaxation}

Application of the dual mode sorption model, Equation (1), to the sorption isotherm for acetonitrile in cellulose acetate resulted in dual mode sorption parameters of $k_{D}=0.282 \mathrm{~g}_{\text {nitrile }} / \mathrm{g}_{\text {polymer }}$, $C_{H}^{\prime}=0.030 \mathrm{~g}_{\text {nitrile }} / \mathrm{g}_{\text {polymer }}$ and $\mathrm{b}=38.1$ (see original paper). Assuming local equilibrium, Equations (6) and (7), were used to fit the FTIR-ATR absorbance data for sorption kinetics from triplicate experiments, using $D_{D}$ as the only adjustable parameter and $F$ set equal to zero. The result for one experiment at activity 0.2 is shown in Figure 1(a). The diffusion coefficients are listed in Table 1 for all activities. For the purposes of comparison, the full model with local equilibrium relaxed, applied to the same experiment at activity 0.2 , is shown in Figure 1(b) and the resulting diffusion coefficients are also listed in Table 1 ( $k_{r}$ fixed at the average value obtained from activity 0.2 ). More detail on how the fits were obtained is given in the original paper.

Given that the diffusion coefficients at a particular activity for the two models are nearly identical, it is worth examining the parameters from local equilibrium relaxation in terms of time scales for diffusion and hole filling (or emptying). The dimensionless grouping, $k_{r} L^{2} / D_{D}$, represents the ratio of diffusion time to hole emptying time. If this group is very large (approaching infinity), then the kinetics of hole filling and emptying can be taken as instantaneous and local equilibrium is approximated. For the experiment shown in Figure $1, k_{r} L^{2} / D_{D}$ is equal to 184 . For all of the experiments, this ratio varied from 64 to 225 . In other words, diffusion is roughly two orders of magnitude slower than hole emptying, and local equilibrium would likely be a reasonable assumption 
within experimental error. Although we determined the ratio of time scales during the writing of the original paper and thought it high, we erroneously concluded that it was of a magnitude that was necessary to capture the lag in the FTIR-ATR data, given that the incorrect expression for local equilibrium could not.

\section{Optimization Tools}

When the dual mode, local equilibrium relaxation model is applied to the FTIR-ATR absorbance data for activity 0.2 , there are two adjustable parameters, $D_{D}$ and $k_{r}$. The fitting program was written with MATLAB Optimization Toolbox. In the program, each parameter is given an initial guess, a lower limit value (very low) and a higher limit value (very high). The values of the two adjustable parameters are used to calculate the absorbance values at each time point, which is then compared to the corresponding experimental values and the sum of squared error (SSE) is calculated. This process was repeated until the SSE was minimized. Since we had convinced ourselves that the local equilibrium assumption needed to be relaxed to describe this system, we did not question the resulting values of the parameters that emerged and also did not carefully examine the SSE as the values of the parameters are varied. Figure 2 plots the SSE as a function of $k_{r} L^{2} / D_{D}$ at a fixed value of $D_{D}$ (the value obtained from the optimization routine). In the larger plot, it is clear that above a certain value of $k_{r} L^{2} / D_{D}$ (approximately 100), the SSE does not change significantly, consistent with the physical interpretation inherent with local equilibrium. However, expanding the plot (see inset) shows that a mathematical minimum in SSE exists, which led to the erroneous conclusion that the resulting parameter value had physical relevance.

\section{Conclusion}

FTIR-ATR spectroscopy remains a powerful tool for studying diffusion in polymers. In glassy polymers, it is able to illustrate that simple Fickian diffusion cannot describe local concentration data as a function of time even though mass uptake can be described with the simpler approach. Introducing dual mode sorption into the transport model captures the anomaly and the technique can be used to validate critical assumptions introduced, such as local equilibrium. 
(a)

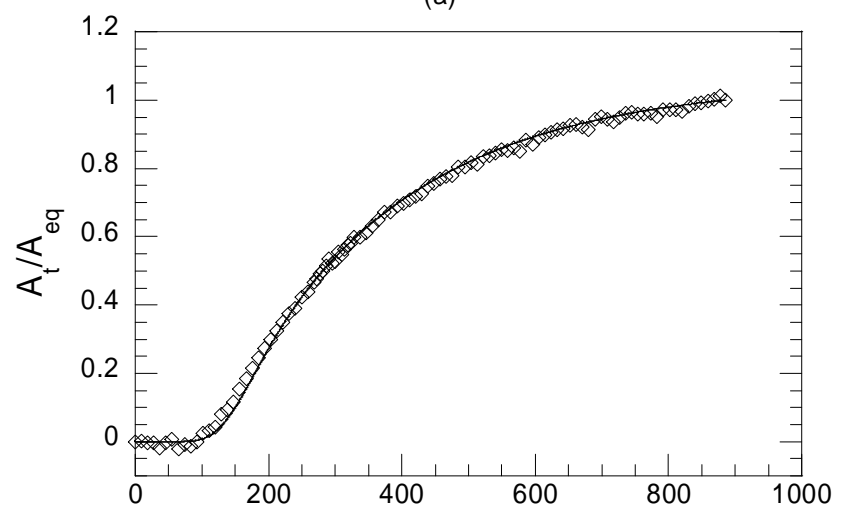

(b)

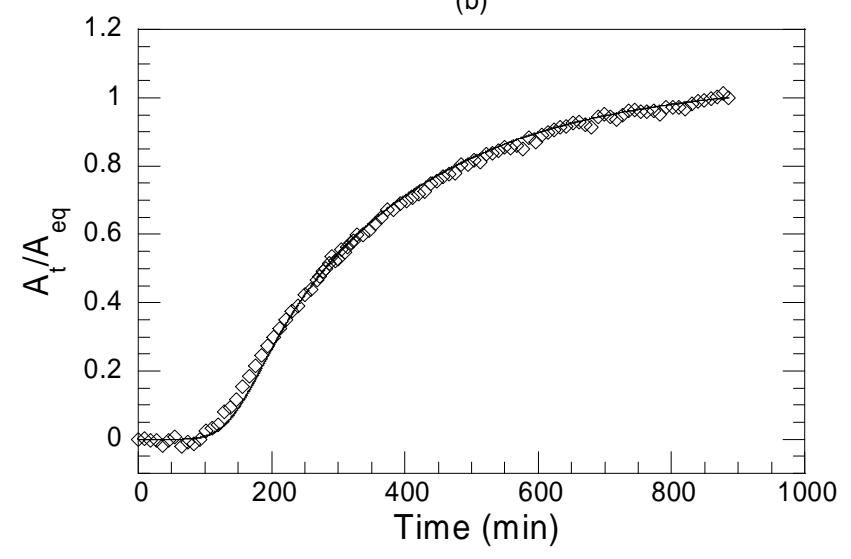

Figure 1. FTIR-ATR absorbance data of acetonitrile in cellulose acetate at activity 0.2 and $\mathrm{T}=25^{\circ} \mathrm{C}$ with (a) the best fit using the dual mode model under local equilibrium assumption $\left(D_{D}=6.67 \times 10^{-10} \mathrm{~cm}^{2} / \mathrm{s}\right)$, and (b) the best fit using the dual mode model with the local equilibrium assumption relaxed $\left(D_{D}=6.34\right.$ $\times 10^{-10} \mathrm{~cm}^{2} / \mathrm{s}$ and $\left.k_{r}=4.48 \times 10^{-3} \mathrm{~s}^{-1}\right) . \mathrm{L}=49.2 \mu \mathrm{m}$. 


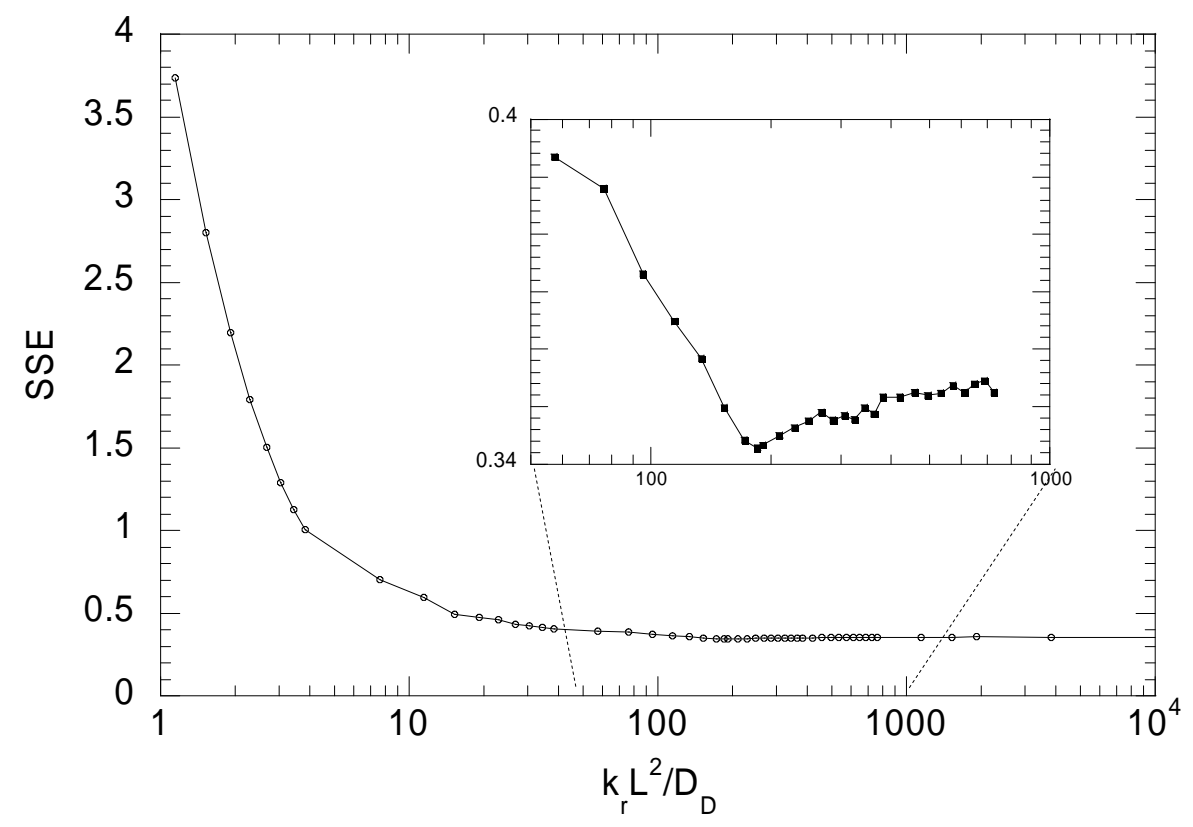

Figure 2. The sum of squared error (SSE) as a function of the ratio of diffusion time to hole emptying time $\left(k_{r} L^{2} / D_{D}\right)$ for the dual mode, local equilibrium relaxation model applied to the experimental data in Figure 1. $D_{D}=6.34 \times 10^{-10} \mathrm{~cm}^{2} / \mathrm{s} . \mathrm{L}=49.2 \mu \mathrm{m}$. 


\begin{tabular}{ccc}
\hline & \multicolumn{2}{c}{$D_{D} \times 10^{10} \mathrm{~cm}^{2} / \mathrm{s}$} \\
\cline { 2 - 3 } Activity & Local Equilibrium & Local Equilibrium Relaxed \\
\hline 0.25 & $7.38 \pm 0.90$ & $7.04 \pm 0.11$ \\
0.15 & $6.46 \pm 0.18$ & $6.38 \pm 0.04$ \\
0.10 & $4.58 \pm 0.24$ & $4.66 \pm 0.01$ \\
0.06 & $2.94 \pm 0.13$ & $2.96 \pm 0.02$ \\
\hline
\end{tabular}

Table 1. Diffusion coefficients assuming local equilibrium (Equations 6 and 7) and those relaxing local equilibrium (Equations 2 and 3) with $k_{r}=4.85 \times 10^{-3} \mathrm{~s}^{-1}$ 